\title{
Spray-coated paper supercapacitors
}

\author{
Mehmet Girayhan Say ${ }^{1}$, Robert Brooke ${ }^{2}$, Jesper Edberg $\mathbb{D}^{2}$, Andrea Grimoldi ${ }^{1}$, Dagmawi Belaineh ${ }^{1}$, Isak Engquist $\mathbb{D}^{1,3 凶}$ and \\ Magnus Berggren ${ }^{1,3}$
}

The increasing demands to further electrify and digitalize our society set demands for a green electrical energy storage technology that can be scaled between very small, and heavily distributed electrical energy sources, to very large volumes. Such technology must be compatible with fast-throughput, large-volume and low-cost fabrication processes, such as using printing and coating techniques. Here, we demonstrate a sequential production protocol to fabricate supercapacitors including electrodes based on cellulose nanofibrils (CNF) and the conducting polymer PEDOT:PSS. Thin and lightweight paper electrodes, carbon adhesion layers and the gel electrolyte are fabricated using spray coating, screen printing, and bar coating, respectively. These all solid-state supercapacitors are flexible, mechanically robust and exhibit a low equivalent series resistance $(0.22 \Omega)$, thus resulting in a high power density $\left(\sim 10^{4} \mathrm{~W} / \mathrm{kg}\right)$ energy technology. The supercapacitors are combined and connected to a power management circuit to demonstrate a smart packaging application. This work shows that operational and embedded supercapacitors can be manufactured in a manner to allow for the integration with, for instance smart packaging solutions, thus enabling powered, active internet-of-things (loT) devices in a highly distributed application.

npj Flexible Electronics (2020)4:14; https://doi.org/10.1038/s41528-020-0079-8

\section{INTRODUCTION}

Wearable electronics, smart packaging, and many other loT systems are receiving great interests and there is of course a need to supply electrical power, sometimes at large power peaks, to all these applications ${ }^{1-3}$. Printed flexible sensors, batteries, and nanogenerators can be integrated with silicon-based electronics components to form complete and operational devices such as sensor labels with wireless readout and combined energy harvesting/storage components. The possibility to exclude bulky power sources enables applications such as flexible and thin patches, possible to use in various medical platforms, wearables and in smart packaging applications ${ }^{4-10}$. The future need for flexible, lightweight energy storage solutions therefore demands conformable device configurations, preferably fabricated using active materials that originates from green sources that also are compatible with easy recycling and a green life cycle management ${ }^{11}$.

Energy storage devices based on cellulose and its derivatives have been demonstrated and integrated into flexible electronics ${ }^{12-15}$ in the past. Within this class of energy devices, the interest for supercapacitors are increasing, in part because of the need to generate short-term power peaks from device that are built up from environmentally friendly and lightweight materials, and that can be made from low-cost and scalable fabrication processes ${ }^{16,17}$. In recent years, these demands have resulted in an increased focus on paper-based energy storage devices due to their flexibility, possibility to provide a large internal surface area and to potentially define a green energy device technology. Additionally, a compatibility with printing technologies are desired in order to manufacture cheap and scalable devices for a vast array of applications, ranging from heavily distributed IoT system to very large volume storage system connected to the power grids ${ }^{18,19}$.
In recent years, we have demonstrated a "power paper" concept that combines cellulose nanofibrils (CNF) and the mixed ionelectron conductor poly(3,4-ethylenendioxythiophene): poly(styrenesulfonate) (PEDOT:PSS), providing a nanonetwork for efficient and high-performing supercapacitor electrodes ${ }^{20}$. This material combination can be utilized as the active component in functional inks for the implementation into printing technologies and has been demonstrated as the electrode in paper-based flexible supercapacitors $^{21}$. It has been shown that these power paper electrodes can deliver a high energy density as well as high power density in free-standing electrodes, being $>15 \mu \mathrm{m}$ in thickness, however the performance of thin film $(<5 \mu \mathrm{m})$ printed electrodes with high power density has so far not been investigated ${ }^{21-24}$ in test cells nor in functional devices and applications.

When printing supercapacitors, the active material should be available as inks so that the electrode, separator, and electrolyte layers can be realized using well-established tools such as screen and inkjet printing, bar coating, and spray coating ${ }^{25}$. However, in the current printing fabrication protocols of the supercapacitors, so far developed and studied, screen or stencil printing have been utilized, which are the techniques that do not meet the demands of thin, conformable electrodes over large areas. In order to attenuate the power paper supercapacitor technology, current challenges include the need for inks with higher viscosity, adhesion/delamination issues and post-processing during fabrication $^{26,27}$. Spray-coating is an efficient and industrially mature technology for fast fabrication of dry films of nanomaterials and polymers with controlled thickness, and is a technology which can easily be implemented in functional optoelectronics and electronics applications ${ }^{28-31}$. Spray-coating allows the formation of thin (even ultra-thin), large area, uniform layers of electroactive materials and has been used to deposit e.g. graphene, graphene oxide and carbon nanotubes serving as the electrode material in supercapacitors and batteries ${ }^{32-36}$. Therefore, spray coating was

\footnotetext{
${ }^{1}$ Laboratory of Organic Electronics, Department of Science and Technology, Linköping University, SE-601 74 Norrköping, Sweden. ${ }^{2}$ RISE Research Institutes of Sweden, Bio- and Organic Electronics, Bredgatan 35, SE-602 21 Norrköping, Sweden. ${ }^{3}$ Wallenberg Wood Science Center, ITN, Linköping University, SE-601 74 Norrköping, Sweden. ${ }^{凶}$ email: isak. engquist@liu.se
} 

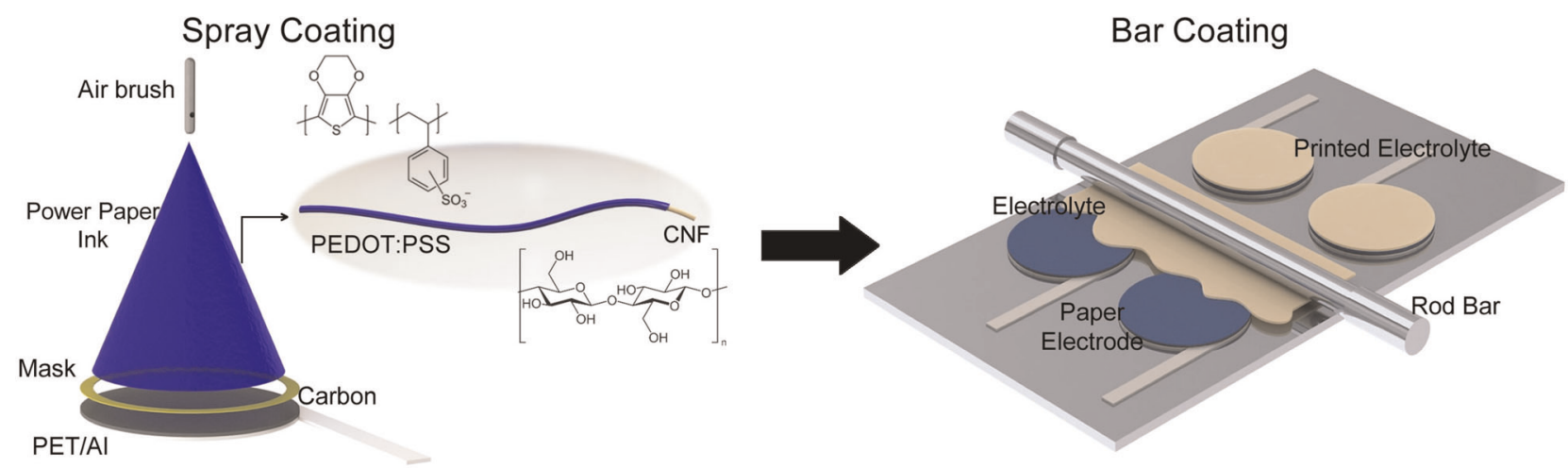

Supercapacitor Assembly
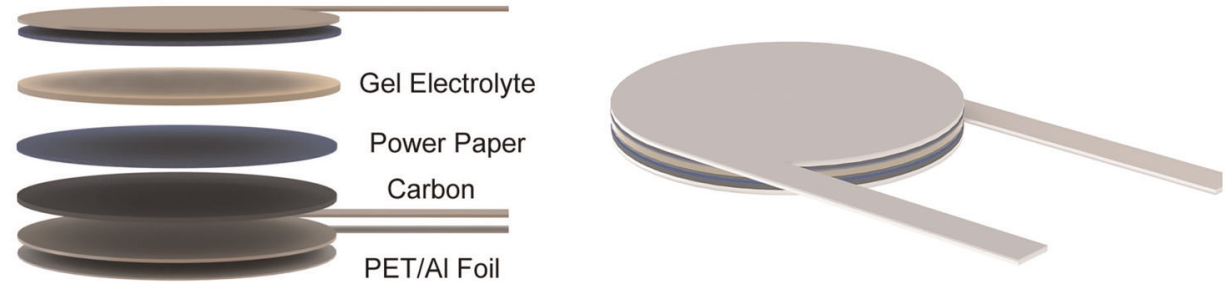

Fig. 1 Fabrication scheme of spray-coated printed paper supercapacitor. Schematics represents the printing steps and components of the paper supercapacitors.

here selected for the fabrication of thin $(1-10 \mu \mathrm{m})$ electroactive films for the realization of electrodes in supercapacitors with high degree of flexibility possible to integrate as slimmed electric energy sources for loT applications, especially targeting smart packaging.

In this work, we investigate the fabrication of CNF-PEDOT:PSS electrodes (hereafter referred to as paper electrodes) using spray coating, and the incorporation of this spraying step into a complete production protocol to produce fully printed supercapacitors. We show that the equivalent series resistance (ESR) of these supercapacitors can be as low as $0.22 \Omega$ by precisely engineer the printing processes. Due to these improvements, high power densities of $\sim 10^{4} \mathrm{~W} / \mathrm{kg}$ can be achieved at high current levels, and capacitances of around $0.1 \mathrm{~F}$ was reached. We have fabricated paper electrodes in the 1-10 $\mu$ m thickness range, while the fully assembled supercapacitor devices measured approximately $140 \mu \mathrm{m}$. The proposed technique holds promise for flexible hybrid electronics for packaging and wearable electronics applications, where mechanical robustness, conformability and fast delivery of energy provided from a small and slim device is desired.

\section{RESULTS}

Supercapacitor fabrication

The fabrication procedure for the printed solid-state paper supercapacitors, as described in the experimental section, is schematically presented in Fig. 1. Before spray coating, the substrate is covered with a mask to control the area of the electroactive material.

The use of a hot substrate during the spraying procedure, makes the main part of the water to evaporate within a couple of seconds, and forces the PEDOT: PSS-CNF to swiftly adhere onto the carbon layer. Initial experiments show that a temperature of $90^{\circ} \mathrm{C}$ is enough to limit agglomeration of the sprayed cellulose fibrils and then helps us to form uniform electrode films. Additionally, a quick removal of solvent not only improve electrode film properties, it also reduces the spraying pause interval, which thus leads to shorter fabrication time.

In the ink development process, the PEDOT:PSS versus CNF weight ratio was kept constant at 2.65:1. This was motivated from the point of view that this ratio gives the highest mechanical strength and also result in the maximum conductivity ${ }^{20}$. During the spraying procedure, the plasticizer content of the ink is crucial and the generated tension between the ink and the carbon surface can lead to film cracking (Supplementary Fig. 3). To maintain uniform film formation and to prevent cracking of the spray-coated films, the ink formulation was modified as compared to the previous publication ${ }^{20}$, simply by increasing the plasticizer content (glycerol) and also by varying its water content. A full description of the resulting ink formulation is given in the Experimental section. After solving the cracking issues and establishing a stable film formation process, paper electrodes of different well-defined thicknesses were made. By increasing the number of spraying cycles, the thickness increases linearly with respect to the number of cycles (Fig. 2a) and corresponding sheet resistance $\left(R_{\mathrm{s}}\right)$ values are given in Fig. $2 \mathrm{~b}$. We find that the overall conductivity of the paper electrode samples remains constant around $\sim 90 \mathrm{~S} / \mathrm{cm}$ for thicknesses ranging from 0.5 to $2.5 \mu \mathrm{m}$. The spray-coated paper electrode can also easily be peeled off from its substrate. Even a $2.5 \mu \mathrm{m}$ thin power paper electrode provides good conductivity, in fact well enough for proper operation in a simple circuit to light up an LED (Supplementary Fig. 4).

To investigate the relationship between the capacitance value and the thickness of the power paper electrode, devices were fabricated and characterized in a two-electrode configuration. The capacitance of the paper is expected to also vary in a linear fashion with respect to thickness and thus the number of spraying cycles correspondingly. Figure 2c displays CV curves of devices with electrode thickness varying from $2.5 \mu \mathrm{m}(5 \mathrm{ml}$ sprayed ink volume) to $7.6 \mu \mathrm{m}$ ( $15 \mathrm{ml}$ ink), which clearly indicate that the capacitance of the devices increases with the electrode bulk volume. Measurements of the capacitance values at $1.0 \mathrm{~A} / \mathrm{g}$ (Fig. 2d, charge-discharge curves shown in Supplementary Fig. 5) shows an approximate increase from 30 to $102 \mathrm{mF}$ (electrode 
a

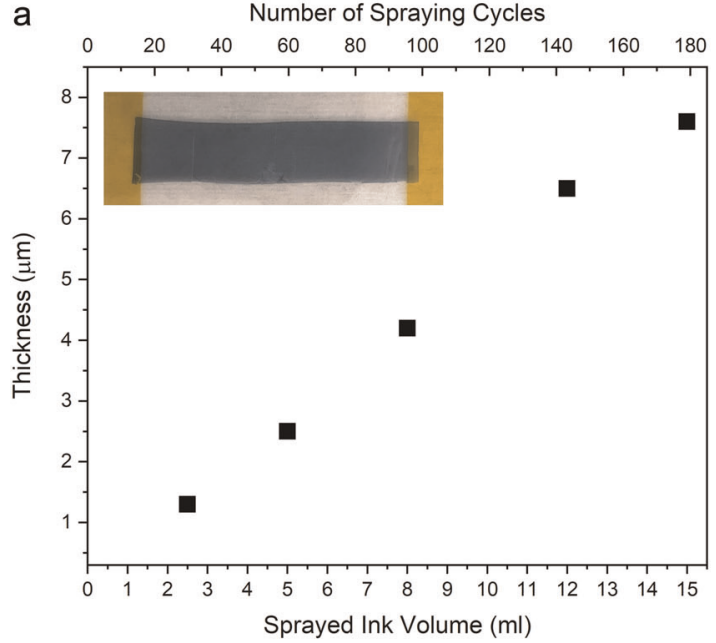

C

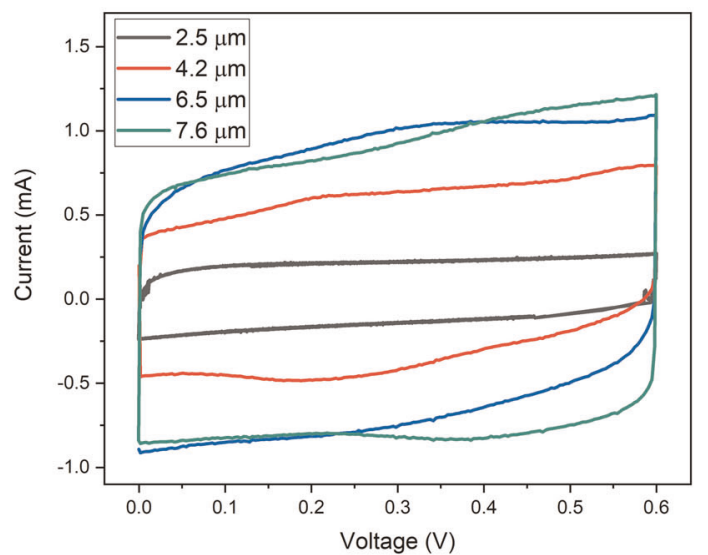

b

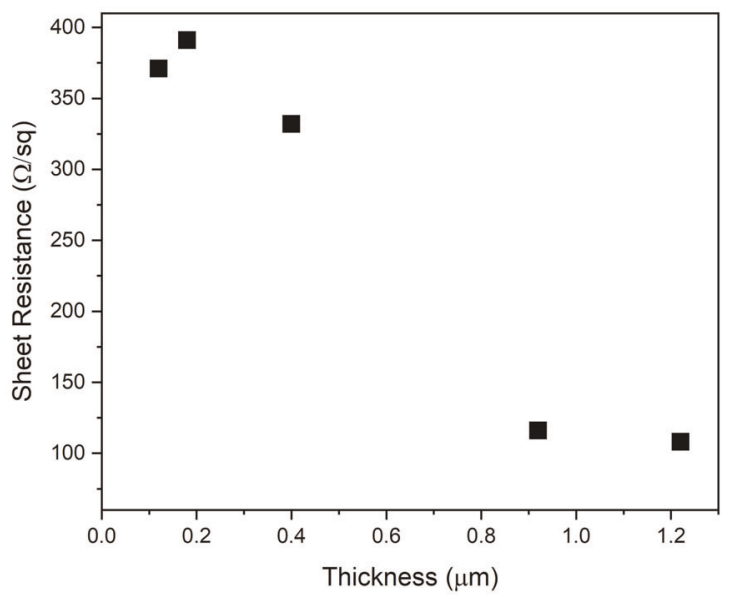

d

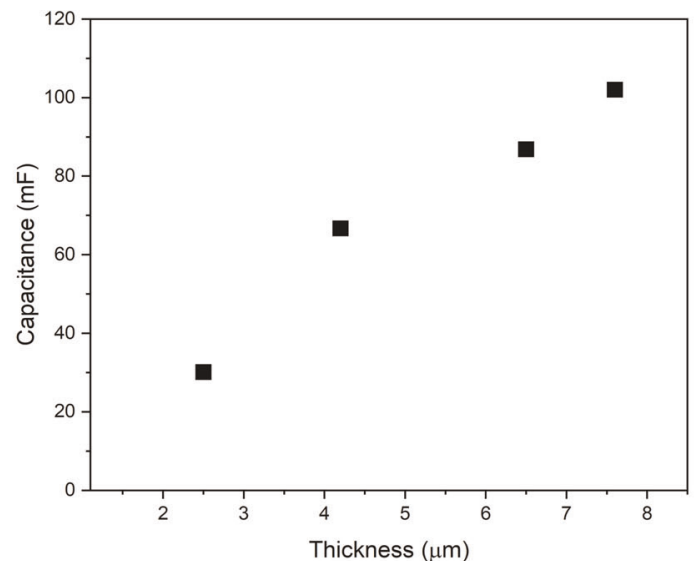

Fig. 2 Paper electrode characterization. a Thickness of the power paper with respect to used ink volume and number of spraying cycles. Inset image shows the $2.5 \mu \mathrm{m}$ sprayed power paper on glass substrate. $\mathbf{b}$ Sheet resistance values of the sprayed power paper electrodes on glass with different thicknesses. c CV curves of printed paper supercapacitors with different ink volumes at a scan rate of $10 \mathrm{mV} / \mathrm{s}$. d Capacitance values of paper supercapacitors with different power paper film thickness.

area of $19.6 \mathrm{~cm}^{2}$ ) when the electrode thickness increases from 2.5 to $7.6 \mu \mathrm{m}$. Since the device capacitance increases in proportion to electrode thickness, we can conclude that the volumetric capacitance remains constant, indicating that charge storage through ion inclusion benefits fully from higher electrode volumes, in other words, higher mass of active material ${ }^{21,37}$.

\section{Supercapacitor performance}

The electrochemical performance of a printed supercapacitor, with a total device thickness of $\sim 140 \mu \mathrm{m}$ (photo and thickness measurement in Supplementary Fig. 6) and that is based on two separated power paper electrodes of $7.6 \mu \mathrm{m}(15 \mathrm{ml}$ sprayed ink volume), is shown in Fig. 3. The CV curves of the supercapacitor were measured for scan rates ranging from 5 to $50 \mathrm{mV} / \mathrm{s}$ and shows almost perfect rectangular shape at each rate, indicating that electrical double layers are formed within the bulk with a low resistive component (Fig. 3a). Galvanostatic charge-discharge (GCD) curves are given in Fig. $3 \mathrm{~b}$ and the resulting capacitance value was calculated and reached $\sim 0.1 \mathrm{~F}\left(5.2 \mathrm{mF} / \mathrm{cm}^{2}, 6.52 \mathrm{~F} / \mathrm{cm}^{3}\right)$ at a current density of $1.0 \mathrm{~A} / \mathrm{g}$. Specific capacitance values, plotted in Fig. 3c, show also quite stable values ranging from 23.1 to $20.1 \mathrm{~F} / \mathrm{g}$ even at very high current densities $(10 \mathrm{~A} / \mathrm{g})$. Additionally, ESR values were calculated from the voltage drop recorded from the GCD analysis. The ESR values are found to reside between 0.22 and $0.27 \Omega$ for $0.1-5.0 \mathrm{~A} / \mathrm{g}$ current densities, suggesting that the device exhibits a rather low internal resistance. These values are quite low while comparing to previous studies of printed supercapacitors ${ }^{38-41}$, typically exhibiting ESR values ranging from 0.55 to $4.0 \Omega$. We tentatively ascribe our low values to a good interfacial contact between the $\mathrm{Al} / \mathrm{C}$ current collector and the spray-coated electrode, thus minimizing interfacial resistance. Additionally, we further investigated our device with electrochemical impedance spectroscopy (EIS) between the frequencies $10 \mathrm{mHz}$ to $100 \mathrm{kHz}$ (Supplementary Fig. 7). The device is limited by mass transport as indicated by the $45^{\circ}$ angle of the Nyquist plot. At low frequency region we see an almost $90^{\circ}$ angle ${ }^{23,24,42-44}$. These two regimes are indicative of finite space diffusion and is often modeled using a so called "open Warburg" element. This behavior is typically observed for mass transport in porous electrodes with a blocking boundary and in energy storage devices ${ }^{45}$.

The printed supercapacitor also showed excellent yield and statistics in terms of the specific capacitance and ESR values recorded from different samples. To further analyze the stability of the devices, we performed GCD cycling tests at $1.0 \mathrm{~A} / \mathrm{g}$. As shown in Fig. 3d, the tested device preserved about $92 \%$ of its capacitance value after 2000 cycles. As shown in the Ragone plot (Fig. 3e), the $140 \mu \mathrm{m}$ thick printed supercapacitor delivers $0.57 \mathrm{Wh} /$ $\mathrm{kg}\left(0.27 \mu \mathrm{Wh} / \mathrm{cm}^{2}\right.$ areal, $0.34 \mathrm{mWh} / \mathrm{cm}^{3}$ volumetric) energy and $22.3 \mathrm{~kW} / \mathrm{kg}\left(10.5 \mathrm{~mW} / \mathrm{cm}^{2}\right.$ areal, $13.4 \mathrm{~W} / \mathrm{cm}^{3}$ volumetric) power 
a

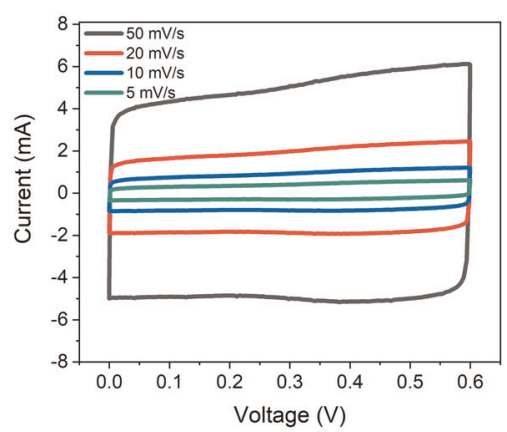

d

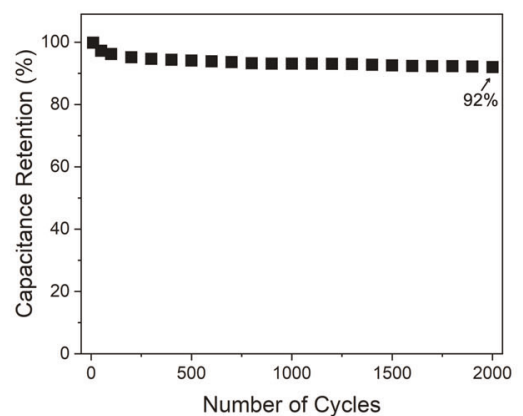

b

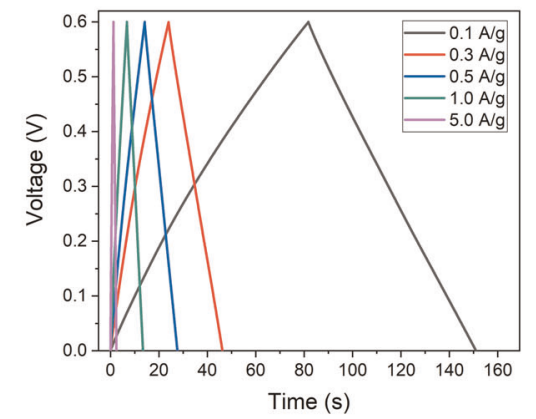

e

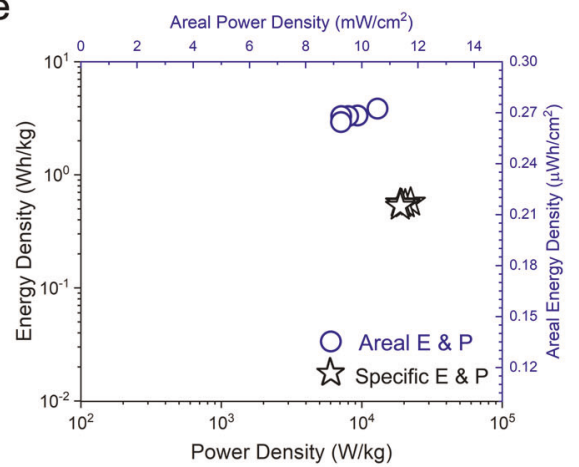

C

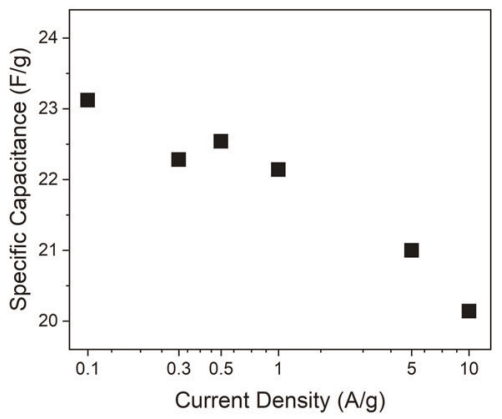

f

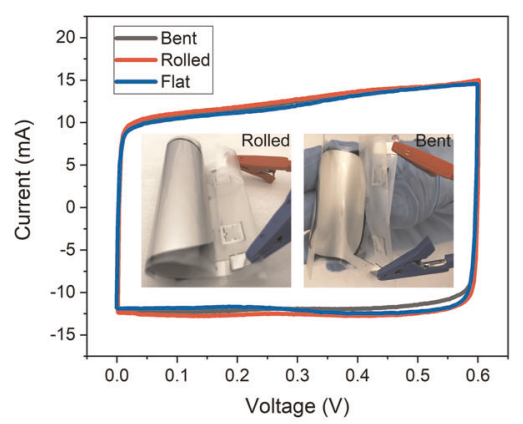

Fig. 3 Electrochemical performance of a $0.1 \mathbf{~ F ~ p r i n t e d ~ s u p e r c a p a c i t o r . ~ a ~} C V$ curves of paper supercapacitors with different scan rates. b Galvanostatic charge-discharge curves at different current densities. c Specific capacitance, (d) cyclic performance, (e) Ragone plot: specific energy and power density performance of an individual supercapacitor, areal (blue circles), specific energy and power density performance (stars) of an individual supercapacitor is displayed in the Ragone plot. f CV curve of the device under different mechanical conditions with the inset showing photos of the device during rolling and bending.

density. These metrics are comparable or even higher (power density) than the previous conductive polymer-paper-based supercapacitors (Supplementary Fig. 8). Although, there are few studies that outperform our devices in terms of energy density, these techniques include polymerization techniques, which limit large scale production and makes it difficult to integrate these electrodes into a flexible cell designs ${ }^{42,43}$. Our solution processed ink approach and implementing sequential printing methods bring mechanical integrity with high power density. In order to demonstrate the performance under mechanical stress, CV curves were also recorded after that the device was rolled and bended, see Fig. 3f. We record more or less identical CV curves at $100 \mathrm{mV} / \mathrm{s}$ regardless if the device is flat, rolled or bent $90^{\circ}$, proving that our printed supercapacitor is functional in application, for which mechanical flexibility and compatibility are important.

\section{Application}

Multiple supercapacitors can be packed together and combined in a small container volume (Supplementary Fig. 9); supercapacitors can also be easily connected in series to increase or set a specific operation voltage. Figure $4 a$ shows two supercapacitors connected in series. GCD and CV of a single supercapacitor and two connected in series are here reported, Fig. $4 b, c$. The doubled voltage is here sufficient to power a dc-dc converter with an output of $3.0 \mathrm{~V}$, which in turn is sufficient for most internet of things and low power electronics applications.

To demonstrate the performance and applicability of coupling supercapacitors with integrated power ICs for smart packaging applications, we designed a dc-dc converter circuitry that was connected to an LED, at the output, then being powered by two serially connected supercapacitors, all residing on a curved substrate (Fig. 4d). Details regarding the circuity and calculations are presented in SI, see Supplementary Figs. 10-12. Supercapacitors were charged up to $1.6 \mathrm{~V}$ at $5 \mathrm{~mA}$ in $12 \mathrm{~s}$ and was thereafter able to deliver a sufficient amount of energy for to turn on the LED via the boost converter. The operation of the LED lasts for $3 \mathrm{~s}$ and here demonstrates a successful hybridization of the printed power paper supercapacitor with different technologies for loT applications in general, here specifically targeting smart packaging application demonstrators. Additionally, our devices with these form factors can be used to store excess energy from wearable energy harvesting technologies to smoothen power fluctuations, power electrochromic emergency indicators or powering an OECT (Organic Electrochemical Transistor) for disposable diagnostic systems.

\section{DISCUSSION}

In this work, we have shown that a conducting polymer-cellulose nanofibril material system perform well when spray-coated to form the two electrodes of a supercapacitor, all produced on a flexible substrate. The complete supercapacitor device was manufactured using a combination of spray coating, screenprinting and bar coating. The resulting fast-production protocol enables us to produce thin, lightweight and fully flexible $0.1 \mathrm{~F}$ supercapacitors on desired areas and planar items. The printed/ spray-coated paper supercapacitors demonstrate a very low series resistance, in fact as low as $0.27 \Omega$ at current density of $5.0 \mathrm{~A} / \mathrm{g}$, a very high power density and overall good stability while experiencing moderate mechanical and bending stress. These combined characteristics make the demonstrated supercapacitors specifically interesting for loT applications in general and for smart packaging in particular, and also for RFID tags, smart wearables and low power sensor systems. 


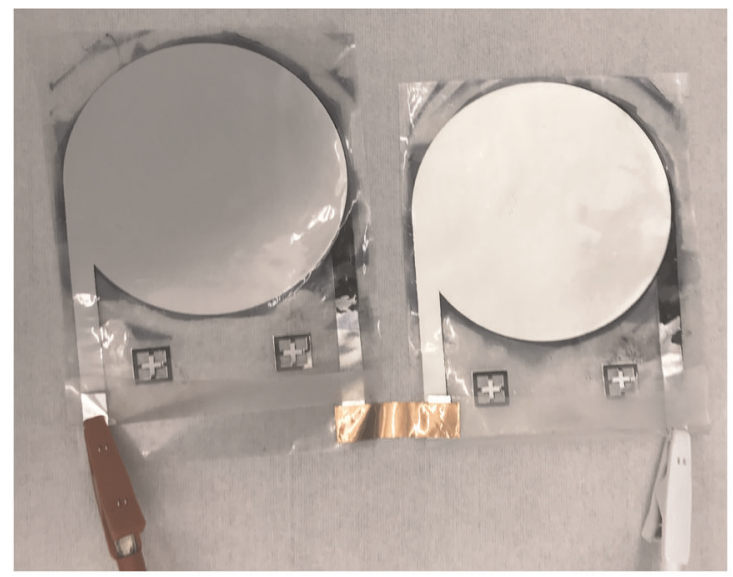

C

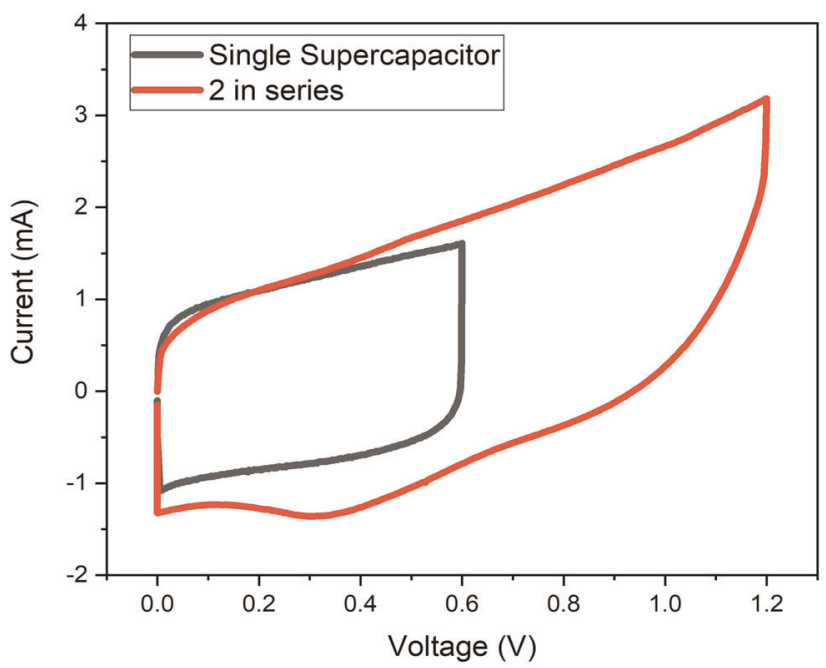

b

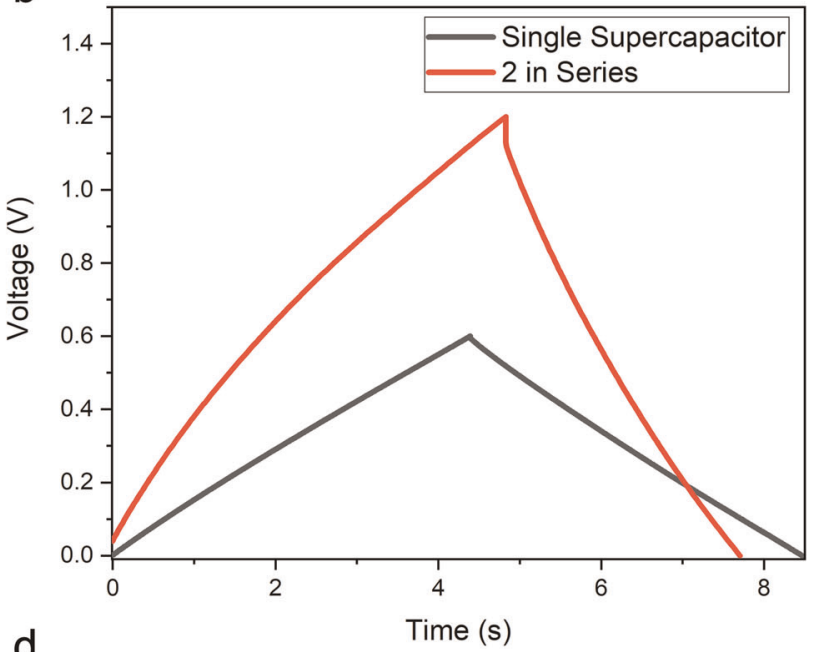

d

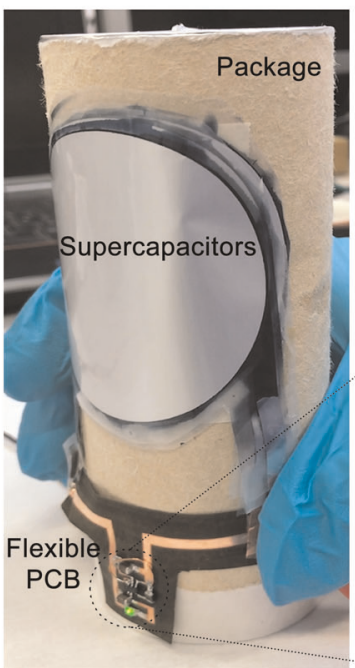

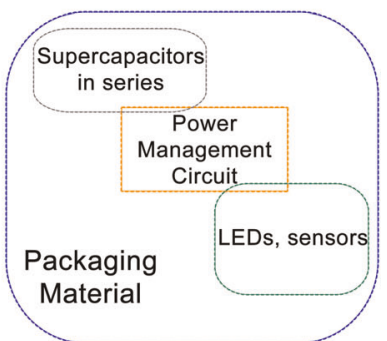

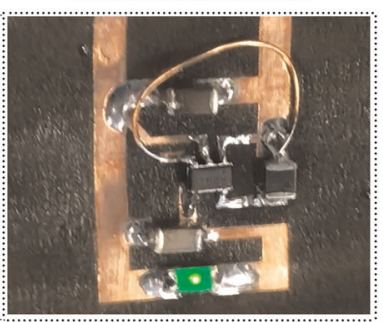

Fig. 4 Applications of the all printed-paper supercapacitors. a Image of two supercapacitors connected in series. $\mathbf{b}$ GCD measurement of two supercapacitor devices connected in series at $1.0 \mathrm{~A} / \mathrm{g}$ current density. c $\mathrm{CV}$ measurement of two series connected devices at a scan rate of $10 \mathrm{mV} / \mathrm{s}$. d Integrated system. LED is connected to boost converter circuit, which is powered by two serially connected supercapacitors on curved packaging material.

\section{METHODS}

Materials

Poly(3,4-ethylenedioxythiophene):poly(styrenesulfonate) (PEDOT:PSS, in the form of the printable ink Clevios PH1000) was purchased from Heraeus Clevios GmbH. Glycerol, Ethylene glycol (EG), 1-ethyl-3methylimidazolium ethyl sulfate $>95 \%$ (EMIM-ES), and Hydroxyethyl cellulose (HEC) were purchased from Sigma-Aldrich. $0.52 \mathrm{wt} \%$ cellulose nanofibrils in water was provided by RISE Bioeconomy. Carbon microcircuit materials 7102 conductive paste ink was purchased from Dupont.

\section{Ink formulation}

Power paper ink containing CNF and PEDOT:PSS was used to form the paper electrode. The $0.52 \mathrm{wt} \%$ cellulose nanofibril dispersion was diluted down to 0.1 wt $\%$ with DI water, and then mixed with PEDOT:PSS, EG and glycerol. The mixture was homogenized with an Ultra-Turrax shear force mixer for 5 min, forming a well dispersed solution with a CNF/PEDOT: PSS/ EG/glycerol ratio of 4.4/11.7/49.4/34.5 (wt\%).

To prepare a bar coating compatible gel electrolyte, EMIM-ES and DI water in a ratio of 1:3.4 were mixed together using magnetic stirring and then placed on a hotplate at $100^{\circ} \mathrm{C}$. $6.2 \mathrm{wt} \% \mathrm{HEC}$ was slowly added. The solution was mixed at $100^{\circ} \mathrm{C}$ for $1 \mathrm{~h}$ to become a gel. Prior to printing, the HEC: EMIM-ES based gel was cooled down to room temperature.
Printing procedures

Prior to spray coating of the power paper ink, a carbon adhesion layer was printed onto $5 \mathrm{~cm}$ diameter dry-phase patterned circular PET/aluminum electrodes (supplied by DPP AB) using a type E2 EKRA screen printing machine. Details regarding this process are given in ref. ${ }^{21}$. The resulting substrate PET/AI/C was placed on a hotplate at $90{ }^{\circ} \mathrm{C}$ for the spraying step. Next, the conducting ink was sprayed in repeated cycles with a commercially available airbrush (Iwata Eclipse, HP-BS, Japan) using nitrogen gas at a pressure of 2.5 bar (Supplementary Fig. 1). The airbrush was positioned $15 \mathrm{~cm}$ above, normal to the substrate and after each spraying cycle (lasting $0.5 \mathrm{~s}$ ), a short pause of $8 \mathrm{~s}$ was added to allow the paper electrode to dry. After the desired number of cycles were completed, the substrate was removed from the hotplate. The gel electrolyte was then deposited directly on the electrode by bar coating using a $100 \mu \mathrm{m}$ diameter rod at a speed of $20 \mathrm{~mm} / \mathrm{s}$ (Supplementary Fig. 2). After drying in air (ambient) to achieve solidification of the gel electrolyte, two identical electrodes were sandwiched together by lamination.

\section{Sheet resistance and conductivity}

In-plane sheet resistance was measured using the four-probe method with a Keithley 2400 Sourcemeter. For this purpose, power paper ink was sprayed on area of $5 \times 20 \mathrm{~mm}$ evenly spaced $(1.2 \mathrm{~mm})$ gold contacts previously evaporated on glass substrates. Several spray cycles at $90^{\circ} \mathrm{C}$ were applied to get the desired film thickness. Sheet resistance values 
were calculated according to the formula:

$\sigma=\frac{1}{R_{\mathrm{s}} \times t}$,

where $\sigma$ is the conductivity, $R_{\mathrm{S}}$ is the sheet resistance, and $t$ is the thickness.

\section{Thickness}

The thickness of sprayed power paper on glass substrates was measured using a Veeco Dektak 3ST surface profile and taking advantage of 10 measurements.

\section{Electrochemical characterization}

Cyclic voltammetry (CV) was performed at scan rates of $5-50 \mathrm{mV} / \mathrm{s}$, galvanostatic charge/discharge measurements (GCD) were performed using $0.1,0.3,0.5,1.0$, and $5.0 \mathrm{~A} / \mathrm{g}$ current density values using a Biologic SP-200 potentiostat in a two-electrode setup.

The capacitance and ESR were calculated from the discharge curves of the galvanostatic measurements using Eq. (2) and Eq. (3), respectively,

Capacitance $(C)=\frac{\Delta t \times I}{V}$,

$\operatorname{ESR}(R)=\frac{V_{\mathrm{d}}}{2 l}$,

where $\Delta t$ is the discharge time, $l$ is the discharge current, $V_{d}$ is the voltage drop at the beginning of the discharging, and $V$ is the difference between operation voltage and $V_{\mathrm{d}}$. Energy density and power density were calculated using Eq. (4) and Eq. (5), respectively.

Energy Density $=\frac{1}{2 m} C V^{2}$,

Power Density $=\frac{V^{2}}{4 m R}$,

where $C$ is the capacitance, $V$ is the operating voltage, $R$ is the ESR, and $m$ is the mass of the electroactive material in one electrode. For areal and volumetric energy and power densities, instead of $m$, electrode area $\left(\mathrm{cm}^{2}\right)$ and volume $\left(\mathrm{cm}^{3}\right)$ is used, respectively.

\section{DATA AVAILABILITY}

The experimental data referenced in this text is available from the authors upon request.

Received: 19 December 2019; Accepted: 29 May 2020;

Published online: 08 July 2020

\section{REFERENCES}

1. Khan, Y. et al. Flexible hybrid electronics: direct interfacing of soft and hard electronics for wearable health monitoring. Adv. Func. Mater. 26, 8764 (2016).

2. Wirthl, D. et al. Instant tough bonding of hydrogels for soft machines and electronics. Sci. Adv. 3, e1700053 (2017).

3. $\mathrm{Pu}, \mathrm{X}$. et al. A Self-charging power unit by integration of a textile triboelectric nanogenerator and a flexible lithium-ion battery for wearable electronics. Adv. Mater. 27, 2472 (2015).

4. Mannoor, M. S. et al. Graphene-based wireless bacteria detection on tooth enamel. Nat. Commun. 3, 763-768 (2012).

5. Kim, J. et al. Miniaturized flexible electronic systems with wireless power and near-field communication capabilities. Adv. Funct. Mater. 25, 4761 (2015).

6. Ostfeld, A. E., Gaikwad, A. M., Khan, Y. \& Arias, A. C. High-performance flexible energy storage and harvesting system for wearable electronics. Sci. Rep. 6, 26122 (2016).

7. Zamarayeva, A. M. et al. Flexible and stretchable power sources for wearable electronics. Sci. Adv. 3, e16202051 (2017).

8. Gao, W. et al. Fully integrated wearable sensor arrays for multiplexed in situ perspiration analysis. Nature 529, 509-514 (2016).

9. Zhong, J. et al. Self-powered human-interactive transparent nanopaper systems. ACS Nano 9, 7399 (2015).

10. Nilsson, $H$. et al. System integration of electronic functions in smart packaging applications IEEE Trans. Compon. Packag. Manuf. Technol. 2, 1723 (2012).
11. Dong, L. et al. Flexible electrodes and supercapacitors for wearable energy storage: a review by category. J. Mater. Chem. A 4, 4659 (2016).

12. Razaq, A., Strømme, M., Nyholm, L. \& Mihranyan, A. Ultrafast all-polymer paperbased batteries. Nano Lett. 9, 3635 (2009).

13. Fraiwan, A., Mukherjee, S., Sundermier, S., Lee, H. \& Choi, S. A paper-based microbial fuel cell: Instant battery for disposable diagnostic devices. Biosens. Bioelectron. 49, 410-414 (2013).

14. Edberg, J., Inganäs, O., Engquist, I. \& Berggren, M. Boosting the capacity of all-organic paper supercapacitors using wood derivatives. J. Mater. Chem. A 1, 145-152 (2018).

15. Kim, J., Kumar, R., Bandodkar, A. J. \& Wang, J. Advanced materials for printed wearable electrochemical devices: a review. Adv. Electron. Mater. 3, 1-15 (2017).

16. Zhang, S. \& Pan, N. Supercapacitors performance evaluation. Adv. Energy Mater. 5, 1-19 (2015).

17. Li, L., Wu, Z., Yuan, S. \& Zhang, X.-B. Advances and challenges for flexible energy storage and conversion devices and systems. Energy Environ. Sci. 7, 2101 (2014).

18. Nyholm, L., Nyström, G., Mihranyan, A. \& Strømme, M. Toward flexible polymer and paper-based energy storage devices. Adv. Mater. 23, 3751-3769 (2011).

19. Tobjörk, D. \& Österbacka, R. Paper electronics. Adv. Mater. 23, 1935-1961 (2011).

20. Malti, A. et al. An organic mixed ion-electron conductor for power electronics. Adv. Sci. 3, 1-9 (2016).

21. Brooke, R. et al. Supercapacitors on demand: all-printed energy storage devices with adaptable design. Flex. Print. Electron. 4, 015006 (2019).

22. Liu, Y. et al. High-performance flexible all-solid-state supercapacitor from large free-standing graphene-PEDOT/PSS films. Sci. Rep. 5, 17045 (2015).

23. Li, Z. et al. Free-standing conducting polymer films for high-performance energy devices. Angew. Chem. Int. Ed. 55, 979 (2016).

24. Li, Y. et al. A strong and highly flexible aramid nanofibers/PEDOT: PSS film for allsolid-state supercapacitors with superior cycling stability. J. Mater. Chem. A 4, 17324-17332 (2016).

25. Sun, J. et al. Printable nanomaterials for the fabrication of high-performance supercapacitors. Nanomaterials 8, 528 (2018).

26. Choi, K.-H., Yoo, J., Lee, C. K. \& Lee, S.-Y. All-inkjet-printed, solid-state flexible supercapacitors on paper. Energy Environ. Sci. 9, 2812-2821 (2016).

27. Liu, L. et al. All-printed solid-state microsupercapacitors derived from selftemplate synthesis of Ag @ PPy nanocomposites. Adv. Mater. Technol. 3, 1700206 (2018).

28. Sandström, A., Asadpoordarvish, A., Enevold, J. \& Edman, L. Spraying light: Ambient-air fabrication of large-area emissive devices on complex-shaped surfaces. Adv. Mater. 26, 4975-4980 (2014).

29. Kramer, I. J. et al. Efficient spray-coated colloidal quantum dot solar cells. Adv. Mater. 27, 116-121 (2015).

30. Kim, S. et al. Purification of PEDOT: PSS by ultrafiltration for highly conductive transparent electrode of all-printed organic devices. Adv. Mater. 28, 10149-10154 (2016).

31. Tenent, R. C. et al. Ultrasmooth, large-area, high-uniformity, conductive transparent single-walled-carbon-nanotube films for photovoltaics produced by ultrasonic spraying. Adv. Mater. 21, 3210-3216 (2009).

32. Huang, C., Young, N. P. \& Grant, P. S. Spray processing of $\mathrm{TiO}_{2}$ nanoparticle/ ionomer coatings on carbon nanotube scaffolds for solid-state supercapacitors. J. Mater. Chem. A 2, 11022-11028 (2014).

33. Wu, Z. S., Liu, Z., Parvez, K., Feng, X. \& Müllen, K. Ultrathin printable graphene supercapacitors with AC line-filtering performance. Adv. Mater. 27, 3669-3675 (2015).

34. Wang, X. et al. A consecutive spray printing strategy to construct and integrate diverse supercapacitors on various substrates. ACS Appl. Mater. Interfaces 9, 28612-28619 (2017).

35. Li, F., Chen, J., Wang, X., Xue, M. \& Chen, G. F. Stretchable supercapacitor with adjustable volumetric capacitance based on 3D interdigital electrodes. Adv. Func. Mater. 25, 4601-4606 (2015).

36. Singh, N. et al. Paintable battery. Sci. Rep. 2, 481 (2012).

37. Cheng, T., Zhang, Y.-Z., Zhang, J.-D., Lai, W.-Y. \& Huang, W. High-performance free-standing PEDOT:PSS electrodes for flexible and transparent all-solid-state supercapacitors. J. Mater. Chem. A 4, 10493-10499 (2016).

38. Zhang, H., Qiao, Y. \& Lu, Z. Fully printed ultraflexible supercapacitor supported by a single-textile substrate. ACS Appl. Mater. Interfaces 8, 32317-32323 (2016).

39. Shao, Y., Wang, H., Zhang, Q. \& Li, Y. Fabrication of large-area and highcrystallinity photoreduced graphene oxide films via reconstructed twodimensional multilayer structures. NPG Asia Mater. 6, e119-e119 (2014).

40. Senokos, E. et al. Large-area, all-solid, and flexible electric double layer capacitors based on CNT fiber electrodes and polymer electrolytes. Adv. Mater. Technol. 2, 1600290 (2017).

41. Guo, Y., Li, W., Yu, H., Perepichka, D. F. \& Meng, H. Flexible asymmetric supercapacitors via spray coating of a new electrochromic donor-acceptor polymer. Adv. Energy Mater. 7, 1601623 (2017).

42. Wang, Z. et al. Surface modified nanocellulose fibers yield conducting polymer-based flexible supercapacitors with enhanced capacitances. ACS Nano 9, 7563-7571 (2015). 
43. Wang, Z. et al. Solution-processed poly(3,4-ethylenedioxythiophene) nanocomposite paper electrodes for high-capacitance flexible supercapacitors. J. Mater. Chem. A 4, 1714 (2016).

44. Anothumakkool, B., Soni, R., Bahange, S. \& Kurungot, S. Novel scalable synthesis of highly conducting and robust PEDOT paper for a high performance flexible solid supercapacitor. Energy Environ. Sci. 8, 1339 (2015).

45. Quang-Nguyen, T. \& Breitkopf, C. Determination of diffusion coefficients using impedance spectroscopy. J. Electrochem. Soc. 165, E826-E831 (2018).

\section{ACKNOWLEDGEMENTS}

The authors would like to thank DPP $A B$ for supplying the patterned current collectors, RISE Bioeconomy for supplying CNF, and the Treesearch research platform access to lab infrastructure. The authors acknowledge Hjalmar Granberg and Anurak Sawatdee for fruitful discussions. Financing was provided from the Swedish foundation for strategic research, Knut and Alice Wallenberg Foundation (Wallenberg Wood Science Center) and the Önnesjö foundation. Open access funding provided by Linköping University.

\section{AUTHOR CONTRIBUTIONS}

I.E. and M.B. designed and planned the project. M.G.S. designed the experiments and carried out the fabrication and characterization of the devices. R.B., J.E., A.G., and D.B. assisted with the experimental procedures, carried out experimental analysis and validation of data. All authors contributed to writing the manuscript.

\section{COMPETING INTERESTS}

The authors declare no competing interests.

\section{ADDITIONAL INFORMATION}

Supplementary information is available for this paper at https://doi.org/10.1038/ s41528-020-0079-8.

Correspondence and requests for materials should be addressed to I.E.

Reprints and permission information is available at http://www.nature.com/ reprints

Publisher's note Springer Nature remains neutral with regard to jurisdictional claims in published maps and institutional affiliations.

Open Access This article is licensed under a Creative Commons Attribution 4.0 International License, which permits use, sharing, adaptation, distribution and reproduction in any medium or format, as long as you give appropriate credit to the original author(s) and the source, provide a link to the Creative Commons license, and indicate if changes were made. The images or other third party material in this article are included in the article's Creative Commons license, unless indicated otherwise in a credit line to the material. If material is not included in the article's Creative Commons license and your intended use is not permitted by statutory regulation or exceeds the permitted use, you will need to obtain permission directly from the copyright holder. To view a copy of this license, visit http://creativecommons. org/licenses/by/4.0/.

(c) The Author(s) 2020 